\title{
Enhancing Physicality in Touch Interaction with Programmable Friction
}

\author{
Vincent Lévesque ${ }^{1}$, Louise Oram ${ }^{1}$, Karon MacLean ${ }^{1}$, Andy Cockburn $^{2}$, \\ Nicholas D. Marchuk ${ }^{3}$, Dan Johnson ${ }^{3}$, J. Edward Colgate ${ }^{3}$, Michael A. Peshkin ${ }^{3}$ \\ ${ }^{1}$ University of British Columbia ${ }^{2}$ University of Canterbury \\ Vancouver, BC Canada Christchurch, New Zealand \\ \{vlev,olouise,maclean\}@cs.ubc.ca andy@cosc.canterbury.ac.nz \{colgate,peshkin\}@northwestern.edu
}

\begin{abstract}
Touch interactions have refreshed some of the 'glowing enthusiasm' of thirty years ago for direct manipulation interfaces. However, today's touch technologies, whose interactions are supported by graphics, sounds or crude clicks, have a tactile sameness and gaps in usability. We use a Large Area Tactile Pattern Display (LATPaD) to examine design possibilities and outcomes when touch interactions are enhanced with variable surface friction. In a series of four studies, we first confirm that variable friction gives significant performance advantages in low-level targeting activities. We then explore the design space of variable friction interface controls and assess user reactions. Most importantly, we demonstrate that variable friction can have a positive impact on the enjoyment, engagement and sense of realism experienced by users of touch interfaces.
\end{abstract}

\section{Author Keywords}

Haptics, tactile feedback, touch screen.

ACM Classification Keywords

H5.2. [User Interfaces]: Interaction Styles, Haptic I/O.

\section{General Terms \\ Design, Experimentation, Human Factors, Performance.}

\section{INTRODUCTION}

As recognition of the impact of emotion on design grows [19], designers seek natural, realistic and organic [26] means of interaction. In 1983, Shneiderman [29] observed the 'glowing enthusiasm' resulting from graphical user interfaces that allowed users to directly manipulate objects. The iPhone's success suggests a similar role of engagement and delight, presumably through the directness and realism of touch interfaces. This is driving renewed research interest in interaction metaphors using touch (e.g. [4,12]).

However, touch interactions with most current devices are 'flat' - all interface objects still feel like the same plastic or

Permission to make digital or hard copies of all or part of this work for personal or classroom use is granted without fee provided that copies are not made or distributed for profit or commercial advantage and that copies bear this notice and the full citation on the first page. To copy otherwise, or republish, to post on servers or to redistribute to lists, requires prior specific permission and/or a fee.

CHI 2011, May 7-12, 2011, Vancouver, BC, Canada.

Copyright 2011 ACM 978-1-4503-0267-8/11/05...\$10.00. glass, so any physical realism must be communicated through visual and auditory illusions. Tactile effects are generally limited to clicks and buzzes that can convey a great deal of information [16] but lack realism.

This paper examines design possibilities and outcomes when touch interactions are enhanced with variable surface friction. We use a Large Area Tactile Pattern Display (LATPaD) [17,32] which creates a friction-reducing 'squeeze film' of air on a touch sensitive display's surface through imperceptible high-frequency vibrations (Figure 1). The prototype consists of a glass plate with bonded piezoelectric actuators atop an LCD screen. A 57x76 mm touchscreen is created with laser-based measurement of finger position. Friction effects are produced by varying vibration amplitude in response to finger movements.

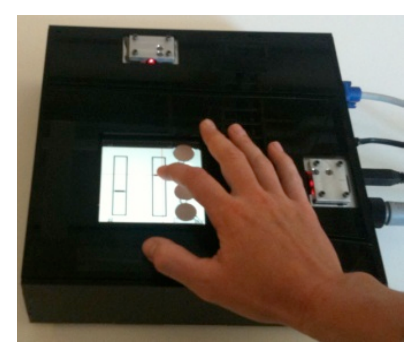

(a)

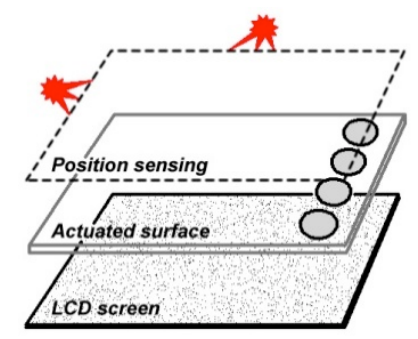

(b)
Figure 1. (a) Picture and (b) illustration of components of the Large Area Tactile Pattern Display (LATPaD).

The ability to vary friction raises interaction possibilities which are interesting from both performance and emotional standpoints. Activities such as pointing and dragging may become more efficient: high friction objects might 'grab' the finger, reducing overshoot and errors, while low friction surfaces should ease sliding movements and reduce finger judder. Emotionally, variable friction may increase perceived realism and subjective satisfaction. In this paper, we report experiments measuring both effects. The specific contributions of our work are as follows:

1. Performance data showing that variable friction can improve performance in touch interactions;

2. Qualitative and quantitative evidence that friction enhanced widgets can positively impact users' emotional response to touch interactions;

3. An exploration of friction-enhanced interface design. 
After providing background, we describe two studies (S1-2) examining the impact of variable friction on target selection performance, without and with surrounding distracters; S3 characterizes the effect of friction on biomechanical control. We then present design concepts exploiting variable friction and examine users' subjective responses (S4). Finally, we discuss our findings and conclude.

\section{BACKGROUND}

Issues grounding our approach range from past work to our own hypotheses on the value of variable friction.

\section{Touch Interaction Without Tactile Feedback}

Buxton et al.'s 1985 analysis of touchscreen limitations still holds: signaling while pointing requires pressure and virtual widgets need haptics [5]. Recent systems have exploited non-feedback touch to address issues like 'fat finger' occlusion, accuracy and the need to feel edges. Roudaut et al. identified these concerns for target acquisition on small touchscreens and proposed zoom techniques for thumbbased selection [27]. Physical metaphors have inspired new, more fluid gestures [25]. Others have elaborated strategies for particular control actions: Potter et al. attributed high accuracy of 'lift-off' selection to its continuous nature [22]. Increasing the tactile feedback available during this contact stream could be even more beneficial.

\section{Tactility in Mobile Devices and Touchscreens}

There are now many examples of interaction design for tactile feedback in touch-surface devices, e.g. [15,23], as well as research and commercial instantiations based on technologies such as piezo- or solenoid-actuated screens. Nearly all rely on vibration. An interesting alternative is electrovibration, which produces sensations of friction or vibration using periodic electrostatic forces [3]. Vibrations can also be applied with passive [24] or actuated pens [10].

While most efforts have layered tactile feedback atop a normal GUI (e.g. tactile overlays on soft keyboards that indicate key proximity and presses [13]), it is arguable that greater benefits are possible for interactions designed around taction. Pokespace relies on forces for its gesture set, and found reduced visual demand in augmented widgets tested in a similar manner to our Study 4 [30].

Generally, speed and accuracy have improved when haptics is included in pointing tasks $[1,6,7]$. This result is nuanced, however: in absence of knowledge of user's destination, feedback may also be encountered for non-target elements, which can introduce obstructions and slowdowns $[6,14,20]$. Furthermore, user preference does not always follow targeting utility [31].

\section{Theoretical Arguments for Variable Friction}

Illusions can be exploited to improve the performance or immediacy of a passive touch interaction. Synchronous sound and graphics can suggest absent tactile feedback. Users "feel" auditory clicks [8]; Apple's iPod took this illusion mainstream. But it fails when the earbuds are out, and lacks the useful physical constraint of a real click. Likewise, visuo-haptic effects such as 'sticky widgets', a manipulation of mouse control-display gain, can improve selection performance by curtailing overshoot in the closedloop phase of motion and enlarging the motor space [2]. Variable friction may further improve performance by making the finger actually stick to the target.

Touch interfaces are also subject to the biomechanics of finger sliding on glass, which produces asymmetric stickslip [18]. 'Judder' is greatest in the distal direction ('north') where friction acts to bend the finger, opposed by extensor muscles. Bending reduces contact angle, increasing the force required to maintain movement ('stick'), then the finger springs forward ('slip'). Proximal or sideways dragging is resisted skeletally with a relatively constant contact angle. Lowering friction should reduce judder for even distal movements.

\section{Variable Friction Devices: the LATPaD}

The LATPaD varies the friction felt by the fingertip at the touch surface. Its operating principle, a squeeze film of air produced by $26 \mathrm{kHz}$ piezo-actuated vibrations, lowers the friction coefficient of a glass surface from $\sim 1.0$ to $\sim 0.15$. Unpublished experiments indicate that the just-noticeabledifference in friction is about $30-40 \%$; thus the LATPaD's dynamic range provides several distinguishable friction levels. Other models demonstrate this effect on larger and smaller plates of arbitrary shape and a range of materials.

Still in early development, our prototype has several limitations. The piezo actuation is compact, but the optical position sensing uses a larger housing. The piezos produce audible noise when active. The vibrational mode used produces nodes where friction reduction is weaker (here, 2 narrow strips parallel to the longer screen axis). Development continues, focusing on these issues. Within 23 years, programmable friction is expected to be deployable in a form factor similar to current touchscreens with uniform feedback and no audible noise.

\section{A Design Space for Variable-Friction Touch}

These and other works (e.g. [28]) demonstrate that while entrancing, current touch technology leaves usability gaps: it is hard to accurately point, select and drag, to select and enter text, and to achieve drag \& drop functions. The illusion of physicality, with both its utility and aesthetic, disappears with the withdrawal of image or sound.

In ongoing work, we are defining the design space where variable friction offers value by filling these gaps. This space is structured around dimensions of (1) rendered effects (e.g. impact, edges, stiffness) and (2) communicated information (e.g. selection support and confirmation, functional availability, spatial navigation). It has guided the design of Study 4, by indicating both the extent of the space to be sampled and opportunities within it. 


\section{STUDIES 1-3: FRICTION PSYCHOPHYSICS}

S1-S3 were conducted in one session and studied the effect of variable friction on target selection and finger motion.

\section{Study 1: Target Selection Without Distracters}

S1 concerns the speed and accuracy of target selection with and without variable friction. Based on the theoretical ability of variable friction to halt the finger on target, we hypothesize:

H1. Variable friction across the surface, with high friction over the target, will improve selection speed and accuracy.

A secondary consideration is the overall level of friction during targeting. Any benefit of varying friction could be explained solely by faster inter-target movement caused by the more slippery surface, rather than by differential friction at the target. The study controls for this effect, leading to the second hypothesis:

H2. There will be no significant difference between selection speed and accuracy when using a constant low level and a constant high level of friction.

Selection modality: lift-off. Friction only matters during sliding surface contact. Thus traditional Fitts' Law [9] 'tapping tasks', where most or all movement occurs above the surface, are unlikely to be influenced by friction effects; we therefore analyzed drag-based selections that are issued when the finger lifts off the surface. This is a common modality on touch devices, particularly when targets are small (e.g., sliding text entry on the iPhone). Furthermore, a lift-off selection modality has been seen to be more accurate than others for touch input in some contexts [22].

Direction. We controlled for movement direction (north, south, east and west), with the aim of revealing movement dynamics rather than testing a specific hypothesis.

\section{Procedure}

Participants were given written instructions on how to interact with the LATPaD. They then tried the device for one minute by rubbing a finger across a checkerboard with high and low friction. They were given the exact procedure for each of trials, which consists of the following steps:

1. Initial state. A thin blue 'control' line and a red 'target' line appear on the display.

2. Acquire control line by touching and remaining stationary on it. For any movement off the control line or off the surface, the trial is repeated.

3. Free control line and begin. After holding for 0.2 second, an audible beep is heard and the control line is freed to move. The clock starts on movement.

4. Drag control line over target. The target turns green to confirm the over-target state, and in some conditions friction changes over the target.

5. Lift-off to select by raising the finger off the target. The target turns back to red briefly and the control line disappears until the next trial.
Participants completed 30 training selections with a 36 pixel $(5.62 \mathrm{~mm})$ wide target (data discarded). Ten were completed with each interface condition with the same order of exposure as for experimental trials (below). To reduce any possible influence of the LATPaD's audible sound, participants listened to white noise through Direct Sound Extreme Isolation EX-29 headphones throughout.

Each participant then completed 336 experimental trials (96 discarded) covering three factors: interface, direction, and target width. The three levels of interface were constant high friction $(H F)$, constant low friction $(L F)$ and variable friction $(V F)$. In $H F$ and $L F$, LATPaD oscillations were always turned off or maximally on, respectively. In $V F$, friction was high (LATPaD off) over the target, but low (maximally on) everywhere else. We did not test inverse variable friction (low over the target and high elsewhere) as it does not offer the psychophysical advantages of 'finger trapping' promised by $V F$. The four levels of direction were north $(n)$, south $(s)$, east $(e)$, and west $(w)$; and the four levels of width were $6,12,24$, and 48 pixels $(0.94,1.87$, 3.74 and $7.49 \mathrm{~mm}$ ). The device was physically rotated when changing direction axis $(n / s, e / w)$ so that movement remained within an optimal friction region. Movement amplitude was always 225 pixels $(35.1 \mathrm{~mm})$. The control and target lines were shifted slightly towards the $n$ or $w$ side of the screen to avoid interference from the raised screen rim. Figure 2 shows the interface for north-direction selections for the four widths.

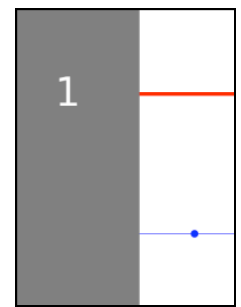

(a)

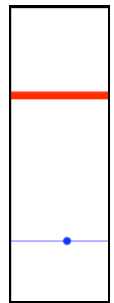

(b)

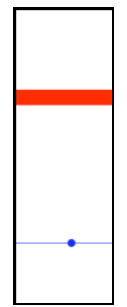

(c)

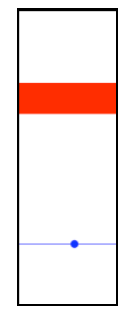

(d)
Figure 2. Target acquisition task for $\mathrm{S} 1$ in the North direction: (a) entire screen for first interface with width of 6 pixels, and partial screen with widths of (b) 12, (c) 24 and (d) 48 pixels.

The experimental trials (target acquisitions) were administered as blocks of 14 trials, each block sharing a direction axis $(n / s, e / w)$, interface level $(L F, H F, V F)$ and target size $(6,12,24,48)$. The first 4 trials of each block were discarded to allow for strategy adaptation. Initial direction was randomized for each block, then alternated on the direction axis. Blocks sharing a direction axis were administered consecutively to minimize physical device rotation; then grouped by interface level to allow questionnaire assessment. Block sets were counterbalanced such that all combinations of $2 !=2$ direction axis orderings and $3 !=6$ interface level orderings were used for one participant. Finally, block target size was randomized within same-interface sets. A total of 24 blocks (2 direction axis $\times 3$ interface levels $\times 4$ target sizes) were administered. 
After each of the 6 sets, participants were asked to comment on the interface used and respond to 5-point Likert-scale questions (strongly disagree to strongly agree): "I performed well / needed to concentrate to accomplish the task / felt confident in my ability to hit the target / felt frustrated / enjoyed interacting with the touchscreen." After completing all trials, participants were asked to rank the interfaces ('ties' permitted) and for final comments. Interface was referred to by order of appearance, reinforced with a numerical label on the side of the display during use.

\section{Participants}

Twelve participants ( 6 female) were recruited from a local university: aged 19-48 (mean 29.4), all right-handed.

\section{Design and Analysis}

Dependent measures are analyzed using a $3 \times 4 \times 4$ repeated measures analysis of variance for the factors interface $\in$ $\{H F, L F, V F\}$, direction $\in\{n, s, e, w\}$, and target width $\in$ $\{6,12,24,48$ pixels $\}$. The dependent measures are selection time, number of errors, time between entering the target and lifting off it, and number of overshoots. We also analyze the goodness of fit to Fitts' Law models (coefficient of determination) and subjective responses.

\section{Results}

In summary: variable friction ( $V F)$ improved targeting performance over $H F$ without compromising accuracy, thus we accept $H 1$. The constant low friction conditions $(L F)$ produced similar results as $H F$, so we also accept $H 2$.

Acquisition time. There was a significant effect of interface $\left(\mathrm{F}_{2,22}=6.89, \mathrm{p}<.01\right)$, with $V F$ fastest (mean $921 \mathrm{~ms}$, s.d. 324), then $H F$ (mean 990, s.d. 344) and $L F$ (mean 1002, s.d. 341); see Figure 3. Posthoc comparison using Bonferroni correction confirms differences between $V F$ and both $H F$ and $L F(\mathrm{p}<.05)$, but not between $H F$ and $L F$.

As anticipated, there was a significant main effect of width $\left(\mathrm{F}_{3,33}=82.2, \mathrm{p}<.001\right)$; but there was also a significant interface $\times$ width interaction $\left(\mathrm{F}_{6,66}=3.85, \mathrm{p}<.01\right)$. Figure 3 suggests that $V F$ performance deteriorated less rapidly across increasing Index of Difficulty than the other conditions. This explanation is supported by the Fitts' Law analysis, which showed strong models for all conditions $\left(R^{2}>0.98\right)$. The lower slope for $V F$ corresponds to an Index of Performance (reciprocal of the slope) of $7.26 \mathrm{bits} / \mathrm{s}$,

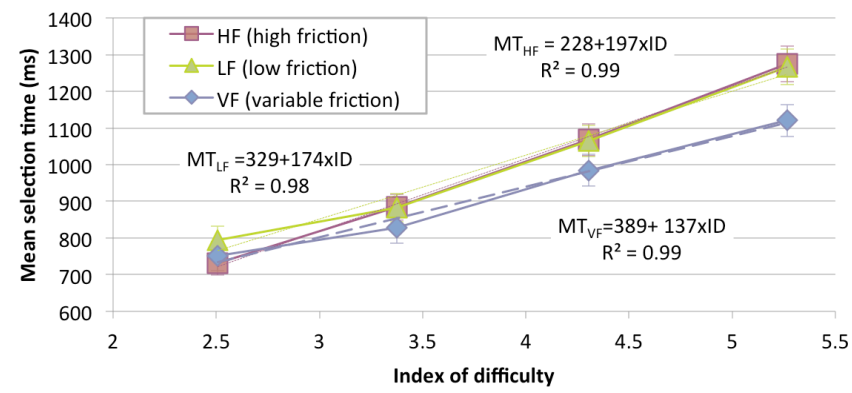

Figure 3. Results and Fitts' law models for interfaces (S1). which is higher than either $H F$ (5.07) or $L F$ (5.74). There was no significant main effect of direction $\left(\mathrm{F}_{3,33}=1.8\right.$, $\mathrm{p}=.17$ ), with means of $943,960,996$ and $984 \mathrm{~ms}$ for $n, s, e$ and $w$ movement respectively.

Accuracy. Analysis of count of trials per block containing an error shows no significant effect of interface $\left(\mathrm{F}_{2,22}=0.74\right.$, $\mathrm{p}=0.49$ ), with similar means of 0.82 errors with $V F$, and 0.7 and 0.81 for $H F$ and $L F$ respectively. The relatively high error rate is due to the use of small targets, and as expected, there is a significant effect of width $\left(\mathrm{F}_{3,33}=53.9, \mathrm{p}<0.001\right)$, with errors increasing from 0.14 errors per block with 48 pixel targets to 1.8 with 6-pixel targets. There was a significant effect of direction $\left(\mathrm{F}_{3,33}=10.0, \mathrm{p}<.01\right)$ with the $s$ movement being the most error prone (1.2 errors per block) and $n$ being the least (0.4). Importantly, however, there was no interface $\times$ width $\quad\left(\mathrm{F}_{6,66}=1.13, \quad \mathrm{p}=0.36\right) \quad$ or interface $\times$ direction interaction $\left(\mathrm{F}_{6,66}=1.16, \mathrm{p}=0.34\right)$.

Source of VF performance advantage. There are several possible explanations for the performance advantage with variable friction: users may move more quickly, resulting in a shorter target approach; they may respond more quickly to the over-target state, resulting in a shorter dwell time over the target; or variable friction may 'trap' the finger on the target, reducing overshoot. To understand which of these are at play, we conducted three more one-way ANOVAs (Figure 4) with dependent variables of approach time (from initial movement to last target border entry), dwell time (from last target border entry to lift-off), and entry count (number of times the target border was entered).

This revealed a significant effect for approach time $\left(\mathrm{F}_{2,22}=5.69, \mathrm{p}<0.05\right)$ with $V F$ faster (mean $634 \mathrm{~ms}$, s.d. 268) than either $H F(690,283)$ or $L F(706,276)$. Neither dwell time $\left(\mathrm{F}_{2,22}=1.0, \mathrm{p}=0.38\right)$ nor entry count $\left(\mathrm{F}_{2,22}=0.87, \mathrm{p}=0.43\right)$ varied significantly, although $V F$ had the lowest mean in both. Consequently, it seems that the largest effect of target acquisition with $V F$ is that it increases users' confidence in moving towards the target, allowing them to approach more quickly without compromising ability to stop abruptly on the target and select it accurately [14].

Subjective results. Participants were asked to rank each interface condition after both $e / w$ and $n / s$ trial blocks. $V F$ was ranked $1^{\text {st }} 58.3 \%$ of the time, $2^{\text {nd }} 29.2 \%$, and $3^{\text {rd }} 12.5 \%$, producing a significant difference (Friedman $\chi^{2}=9.5$, $\mathrm{p}<.01$ ); mean rankings were $1.5,2.0$ and 2.1 for $V F, L F$,

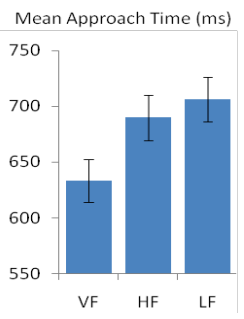

(a)

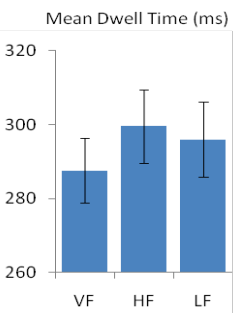

(b)

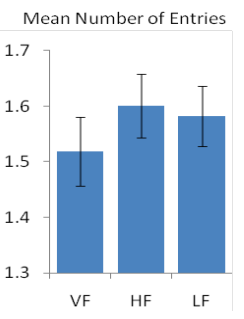

(c)
Figure 4. (a) Approach time, (b) dwell time, and (c) number of target entries as a function of the interface condition (S1). 
and $H F$. Questionnaire responses (Table 1) show that mean ratings for $V F$ were most appreciative in all five questions, but only significantly so for enjoyment.

\begin{tabular}{l|c|c|c|c|c}
\multicolumn{1}{c}{} & \multicolumn{1}{c}{ VF } & \multicolumn{1}{c}{ LF } & \multicolumn{1}{c}{ HF } & \multicolumn{1}{c}{$\chi_{r}^{2}$} & \multicolumn{1}{c}{ Sig } \\
\hline Performance & $3.9(0.7)$ & $3.7(0.8)$ & $3.5(0.7)$ & 2.3 & $=0.31$ \\
Concentration & $3.2(1.0)$ & $3.7(1.0)$ & $3.5(1.0)$ & 3.4 & $=0.18$ \\
\hline Confidence & $4.0(0.7)$ & $3.5(0.8)$ & $3.7(0.6)$ & 2.8 & $=0.25$ \\
Frustration & $1.8(0.7)$ & $2.0(0.8)$ & $2.2(0.8)$ & 2.3 & $=0.31$ \\
\hline Enjoyment & $4.2(0.7)$ & $3.8(0.8)$ & $3.6(0.8)$ & 5.8 & $=0.05$ \\
\hline \hline
\end{tabular}

Table 1. Mean (st. dev.) questionnaire responses, with $1=$ strongly disagree, and 5 = strongly agree (S1).

\section{Study 2: Target Selection With Distracters}

Previous work has demonstrated that tactile feedback can negatively influence performance in the presence of distracter targets [6]. This is a critical limitation, as most practical deployments will involve distracters. Therefore we tested the hypothesis that:

H3. Variable friction will not adversely affect targeting performance in the presence of distracter targets.

\section{Procedure, Apparatus and Participants}

The 12 participants from S1 proceeded immediately to S2. The task was identical to S1 but the space from the control line to the target and beyond was populated with distracters of the target's width. All distracters produced the same visual and tactile effects as the target, i.e. highlighted green to indicate over-target state, and presence of friction effects. Distracters were otherwise black.

Distracter density is an important variable for $H 3$, so three levels of distracter separation were used: 5, 20, and 40 pixels (Figure 5). Targets and distracters were 24 pixels in width. The number of distracters placed before the target varied from 1 to 3 ; one distracter was always behind the target. We tested only one direction axis, and selected $n / s$ for a greater range of biomechanical effects.

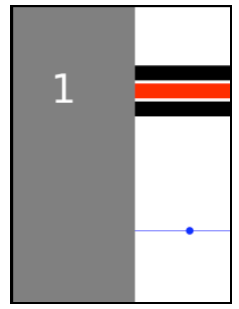

(a)

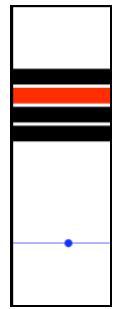

(b)

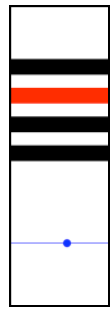

(c)

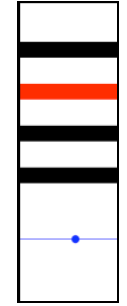

(d)

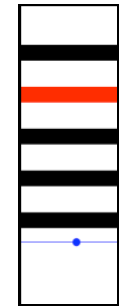

(e)
Figure 5. Target acquisition task for $\mathrm{S} 2$ in the $\boldsymbol{n}$ direction: (a) entire and (b-e) partial screen for first interface with 1 to 3 pre-target distracters and a separation of 5, 20 and 40 pixels.

A block held twenty-two target selections (trials); three blocks were performed per interface condition. The direction $(n, s)$ was initially randomized for each block and then alternated. The number of distracters and their spacing were selected randomly for the first four trials (discarded). To prevent memorization of the tactile pattern leading to the target and its use as an aid, the remaining 18 trials cycled randomly through all $3 \times 3$ combinations of distracter number and spacing in each direction $(n, s)$. Interface order and subjective responses were controlled as for $\mathrm{S} 1$.

\section{Design}

The same dependent measures as $\mathrm{S} 1$ are analyzed in a $3 \times 2 \times 3 \times 3$ repeated measures analysis of variance for interface $\in\{H F, L F, V F\}$, direction $\in\{n, s\}$, distracter spacing $\in\{5,20,40$ pixels $\}$ and number $\in\{1,2,3\}$.

\section{Results}

In summary: the results show no effects of interface (main or interactions) for dependent measures of time or errors, thus we accept $H 3$.

Performance. A four-way ANOVA showed a significant effect of distracter spacing on acquisition time $\left(\mathrm{F}_{2,22}=13.3\right.$, $\mathrm{p}<.01$ ), with mean of 908,892 and $874 \mathrm{~ms}$ for 5, 20 and 40 pixel spacing respectively. There were no significant main effects or interactions involving interface for either task time or errors. Mean times were similar $(900,892,882 \mathrm{~ms}$ for $\left.V F, H F, L F ; \mathrm{F}_{2,22}=0.26, \mathrm{p}=0.78\right)$, as were per block error rates $\left(0.16,0.22,0.19 ; \mathrm{F}_{2,22}=0.71, \mathrm{p}=0.5\right.$ ). $\mathrm{S} 1$ trials (zero distracters) with $n / s$ directions and 24-pixel targets were also compared to $\mathrm{S} 2$ (1, 2 or 3 distracters). A three-way ANOVA for factors interface, direction and number of distracters revealed no significant effects.

Subjective results. The participant's interface rankings were similar to $\mathrm{S} 1$ : a significant preference for $V F$, but nonsignificant responses to other questions.

\section{Study 3: Constant Velocity Dragging}

To characterize physical effects occurring at friction borders, we conducted a third shorter study with the same participants immediately after S2. Participants tried to achieve a target drag velocity in repeated bidirectional strokes across the display by following an audible metronomic tempo. We tested two speeds $(50 / 100 \mathrm{~mm} / \mathrm{s}$, or $320.5 / 641.0$ pixels $/ \mathrm{s})$, and both orientations $(n / s, e / w)$. Friction patterns (not shown visually) were produced in the middle of the strokes, including constant levels, step changes, and single or sequential pulses with the same extent, number and separation as S1 and S2's targets and distracters. Each participant performed 168 trials in six blocks. The first six trials of each block were discarded to allow speed adjustment. The order of patterns was randomized within blocks and each pattern shown once.

\section{Results}

Finger position and velocity trajectories were plotted against friction state and inspected visually for all 1584 trials. An effect of variable friction is clear in many trajectories. Figure 6 shows the best examples, where a step increase or decrease in friction leads to a temporary deceleration or acceleration of the finger. This suggests that finger velocity is affected at least under certain conditions. A sticky target with variable friction may therefore be truly sticky. This effect may in other cases have been reduced by 
stick-slip of the fingerpad or finger pressure, or masked by the low spatio-temporal data. A quantitative analysis is beyond the scope of this paper, but currently underway.

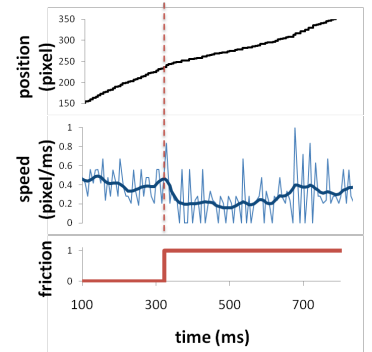

(a)

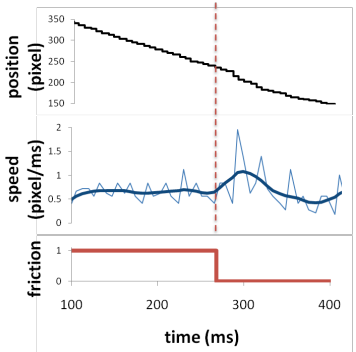

(b)

Figure 6. Selected $\mathrm{S3}$ results: (a) deceleration after increase in friction $(\mathrm{P} 2, e),(b)$ acceleration after step decrease $(\mathrm{P} 4, n)$.

STUDY 4: DESIGN EXAMPLES AND USER EXPERIENCE We explored interface design for variable friction interfaces in an iterative process, beginning with glass etched prototypes, then exemplar designs, which finally led to a study to establish their emotional and subjective impact.

\section{Demonstration Applications and Study Tasks}

Four exemplar widgets were designed to provide good coverage of tactile sensations and of communication functions that variable friction might support.

Alarm Clock. Users set the alarm time using wheel widgets and the alarm sound using a combo box (Figure 7a-c). The wheels produce strong ticks by abruptly increasing friction as items near their center (Figure $7 \mathrm{~d}$ ). The combo box produces similar ticks but with friction peaks between targets (Figure 7e). The Alarm Clock study task involved setting the time and sound to a value displayed at the bottom of the screen.

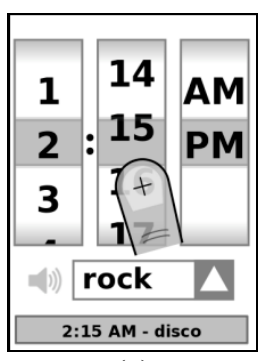

(a)

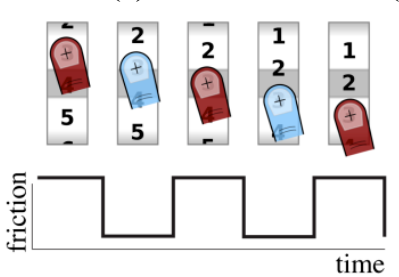

(d)

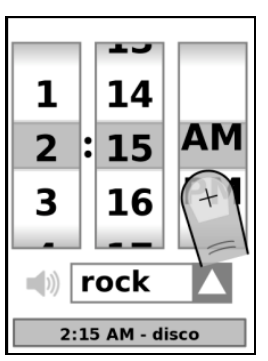

(b)

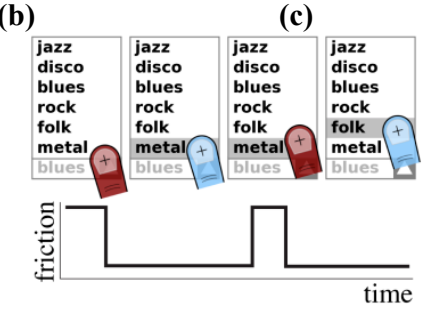

(e)

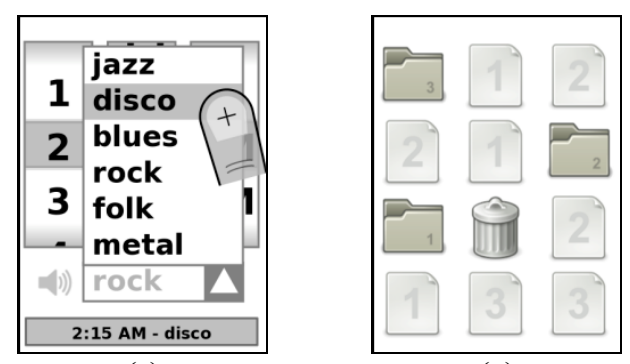

(a)
File Manager. File, folder and recycle bin icons are arranged in a $3 \times 4$ array (Figure $8 \mathrm{a}$ ); target icons enlarge $20 \%$ when a file hovers over them (Figure $8 b-c)$. Initially low, friction increases abruptly over folders and oscillates at $37.5 \mathrm{~Hz}$ over the recycle bin, producing a bump and buzzing respectively (Figure 8d). The File Manager study task involved moving eight files labeled 1 to 3 into folders.

Game. The game consists of bouncing a ball against a round cursor around the finger and breaking bricks (Figure $9 \mathrm{a}-\mathrm{c})$. Some require multiple hits to be broken; others produce special effects - releasing a second ball or making the ball bounce erratically. The ball is launched by compressing a spring, with gradual increase in friction to simulate resistance (Figure 9a, d). For ball impact (Figure $9 \mathrm{~b}, \mathrm{e})$, friction abruptly increases as the ball nears the cursor. Erratic bouncing (Figure 9c) produces the friction oscillation used on the File Manager recycle bin. The Game study task was to play the game, with ten difficulty levels.

Text Editor. Words are selected by dwelling with a cursor extending above the finger, avoiding occlusions. While dragging a word, collisions with adjacent words result in visual compression up to $30 \%$, after which words remain fixed (Figure 10a). A word swaps with its neighbor when the cursor reaches a position where it can be relocated. Swiping left or right flips pages (Figure 10b). When dragging within a line, friction increases with compression, and drops abruptly after a swap, creating a popping sensation (Figure 10c). When dragging between lines, friction effects fade in and out across lines, with a brief friction pulse in-between. Page swaps trigger a tick via an abrupt increase in friction. The Text Editor study task consisted of 'fixing' sentences by reordering words within pages - e.g. "the store grocery sells yellow tomatoes green bananas red lettuce and eggplants purple."

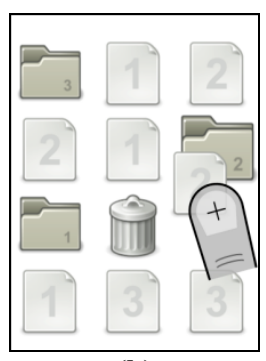

(b)

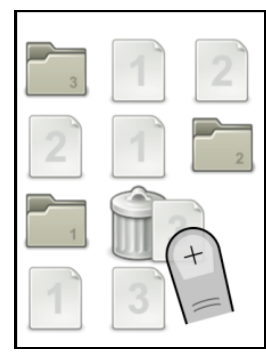

(c)

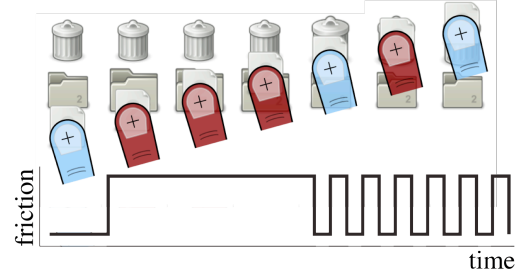

(d)

Figure 8. File Manager: (a) initial screen, moving a file into (b) a folder or (c) recycle bin, and (d) friction patterns while over a folder or bin. The finger color changes from light blue to dark red as friction increases.
AM/PM wheel, (c) sound combo box and friction patterns while selecting (d) hour and (e) sound. The finger color changes from light blue to dark red as friction increases. 


\section{Experimental Procedure}

Participants interacted with each of the four applications twice, with and without variable friction. Each interaction was limited to 2 minutes to provide exposure without boredom. Each application was presented first for $1 / 4$ of the participants, and the order of the other applications was randomized. Half of the participants experienced all applications first with variable friction, the other half without. Participants were instructed to focus on experience rather than performance.

A User Engagement Scale (UE1-10) was used after each condition (twice per application; with and without friction). A tactile feedback questionnaire (TF1-7) was completed after each interaction with variable friction (once per application feature). Once both friction conditions were completed with an application, a comparison questionnaire (C1-5) was administered, followed by a short interview (I13). This procedure was repeated for all four applications, and followed by a final questionnaire (F1-2). The User Engagement Scale used a 7-point Likert scale and all others a 5-point scale (strongly disagree to strongly agree).

The User Engagement Scale (UE1-10) was adapted from a validated 31-question questionnaire developed to assess six aspects of engagement: Focused Attention, Perceived Usability, Aesthetics, Endurability, Novelty and Felt Involvement [21]. Ten of the 31 questions were adapted, spanning all aspects:

UE1. I was absorbed in my interaction task.

UE2. I felt in control of my interactive experience.

UE3. I found this application confusing to use.

UE4. I liked the visual and tactile effects used in this application.

UE5. This application appealed to my visual and tactile senses.

UE6. I would recommend this application to my friends and family.

UE7. I would have continued to interact with this app. out of curiosity.

UE8. I felt interested in my interaction task.

UE9. This interactive experience was fun.

UE10. I felt involved in this interaction task.

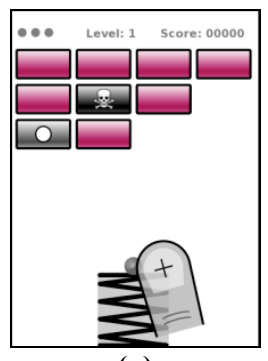

(a)

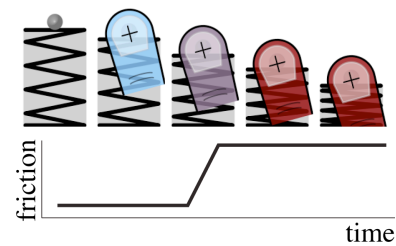

(d)

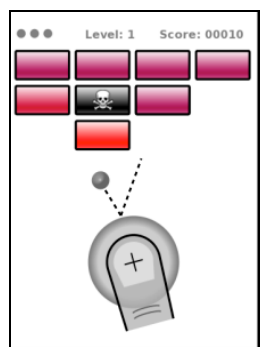

(b)

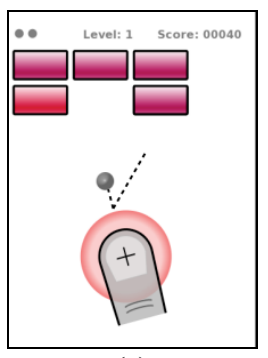

(c)

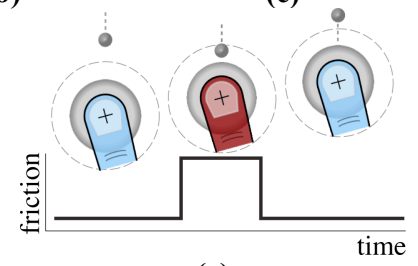

(e)

Figure 9. Game: (a) launch, (b) normal and (c) erratic bounce, and friction patterns during (d) launch and (e) bounce. Low friction shown as a blue finger, high friction red.

The tactile feedback questionnaire (TF1-7) was completed for the main tactile features of each application: hour/minute wheel, AM/PM wheel and sound combo box for Alarm Clock; folders and recycle bin for File Manager; launcher, normal and erratic bounce for Game; movement within or between lines and page swapping for Text Editor. Participants were asked if they noticed the feature, and if so rated whether the tactile feedback was (TF1) weak, (TF2) natural, (TF3) informative, (TF4) annoying, (TF5) matched the visuals, (TF6) felt good, and (TF7) was preferred.

The comparison questionnaire (C1-5) asked if tactile feedback (C1) was preferred, (C2) made the task easier to perform, (C3) the application more enjoyable, (C4) the interface more realistic and (C5) made them more confident. Interview questions (I1-3) asked participants (I1) to describe the sensations, (12) what they liked and didn't like about the tactile feedback, and (I3) how they would improve it. The final questionnaire (F1-2) asked (F1) if participants would turn this type of feedback off on their phone, and (F2) if tactile feedback improved their experience.

\section{Participants}

The data for eight participants were rejected due to protocol irregularities and hardware or software complications that may have affected subjective responses. The remaining twelve participants (6 females) were aged from 19 to 38 (mean 24.3). Eleven were students, only four from engineering and computer science. Ten used touchscreen phones or music players once a week or more. None participated in S1-3.

\section{Results}

The participants' comments and questionnaire responses demonstrate that friction can improve the subjective experience of touch interactions. They also provide insights into potential negative effects, which need to be addressed by design. We begin with interview responses and comments, and then report questionnaire results.

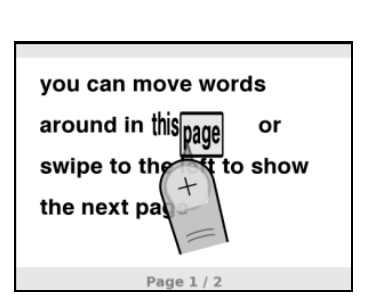

(a)

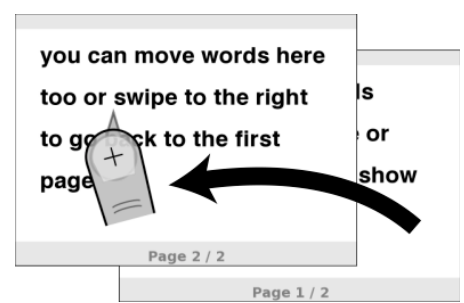

(b)

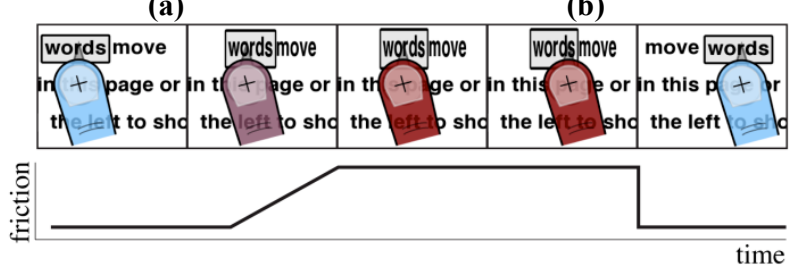

(c)

Figure 10. Text Editor: (a) word movement, (b) page swap and (c) friction patterns while moving a word. The finger color changes from light blue to dark red as friction increases. 
Interview Responses

Several comments show that variable friction enhanced the participant's sense of realism: “When I was moving the words against something, I could feel something squeeze back." (P3, Text Editor); "I knew I was actually touching it." (P2, File Manager); "It feels [...] as if turning the wheels." (P11, Alarm Clock).

Comments also show that variable friction increased awareness of the system state, some suggesting a reduced dependence on vision: "I think it gives me accuracy, [...] if I closed my eyes I would be able to predict the amount of scrolling that I do." (P5, Alarm Clock); "Feel more informed ... when I am moving on the line I can feel each word." (P11, Text Editor); "For the garbage bin it's like oh ah don't do it." (P3, File Manager).

Importantly, several comments showed that participants liked the friction effects: "This is nice... it makes things a lot more interesting." (P3, Game); “I liked the sensation while I am rolling" ( $\mathrm{P} 8$, Alarm Clock).

Nine of the twelve participants were predominantly positive in their comments about variable friction for one (1), two (3), three (4) or four (1) of the applications. The remaining three were predominantly negative or neutral. Negative words used to describe variable friction included "unpleasant", "weird", "creepy", "annoying" and "itchy". The tactile feedback was often described more neutrally using physics-related terms such as "resistance", "friction", "slippery" and "sticky". Negative comments were often aimed at the limitations of the applications but also suggested potential pitfalls of variable friction such as "[getting] in the way of trying to move" (P8, File Manager) or inducing fatigue through overuse (P3, Alarm Clock).

Interestingly, there was little cross-participant consistency in assessing which applications and effects were positive additions. Similarly, participants differed in their assessment of feedback strength, with two stating that stimuli were too weak and one too strong. This suggests a need for friction effects to be very carefully designed and customizable by end users. A majority of participants also spontaneously discussed variable friction's integration in commercial devices and voluntarily shared their impressions for 5-15 minutes after experiment completion.

\section{Questionnaire Responses}

The questionnaire responses tend to amplify the overall positive response to variable friction effects.

User Engagement Scale (Table 2). Responses for variable friction were positive or neutral, except for control (UE2) in Alarm Clock, confusion (UE3) in Alarm Clock and Game, and liking (UE4) in Game; none statistically significant. Of forty comparisons (4 applications $\times 10$ questions), variable friction received better scores in $30: \chi^{2}=9.0, \mathrm{p}<.005$.

Tactile Feedback. Marked differences were found in the noticeability of friction effects, with only $25 \%$ noticing the

\begin{tabular}{|c|c|c|c|c|c|c|c|c|}
\hline & \multicolumn{2}{|c|}{ Clock } & \multicolumn{2}{|c|}{ Files } & \multicolumn{2}{|c|}{ Game } & \multicolumn{2}{|c|}{ Text } \\
\hline & $H F$ & $V F$ & $H F$ & $V F$ & $H F$ & $V F$ & $H F$ & $V F$ \\
\hline $\begin{array}{l}\text { UE1. } \\
\text { Absorbed. }\end{array}$ & $\begin{array}{c}4.7 \\
(1.2)\end{array}$ & $\begin{array}{c}4.9 \\
(0.9)\end{array}$ & $\begin{array}{c}4.8 \\
(1.2)\end{array}$ & $\begin{array}{c}5.4 \\
(1.2)\end{array}$ & $\begin{array}{c}5.6 \\
(0.9)\end{array}$ & $\begin{array}{c}5.8 \\
(0.6)\end{array}$ & $\begin{array}{c}5.1 \\
(0.8)\end{array}$ & $\begin{array}{c}5.1 \\
(1.2)\end{array}$ \\
\hline $\begin{array}{l}\text { UE2. } \\
\text { Control. }\end{array}$ & $\begin{array}{c}5.3 \\
(0.8)\end{array}$ & $\begin{array}{c}5.1 \\
(1.3)\end{array}$ & $\begin{array}{c}5.6 \\
(0.7)\end{array}$ & $\begin{array}{l}5.6 \\
(0.9)\end{array}$ & $\begin{array}{c}4.9 \\
(1.2)\end{array}$ & $\begin{array}{c}5.3 \\
(0.9)\end{array}$ & $\begin{array}{c}4.0 \\
(1.7)\end{array}$ & $\begin{array}{c}4.8 \\
(1.9)\end{array}$ \\
\hline $\begin{array}{l}\text { UE3. } \\
\text { Confusion. }\end{array}$ & $\begin{array}{c}2.0 \\
(1.2)\end{array}$ & $\begin{array}{c}2.3 \\
(1.3)\end{array}$ & $\begin{array}{c}1.7 \\
(0.8)\end{array}$ & $\begin{array}{c}1.7 \\
(0.9)\end{array}$ & $\begin{array}{c}1.8 \\
(0.9)\end{array}$ & $\begin{array}{c}2.2 \\
(1.2)\end{array}$ & $\begin{array}{c}2.5 \\
(1.4)\end{array}$ & $\begin{array}{c}2.3 \\
(1.6)\end{array}$ \\
\hline $\begin{array}{l}\text { UE4. } \\
\text { Liked. }\end{array}$ & $\begin{array}{c}4.4 \\
(1.3)\end{array}$ & $\begin{array}{c}5.0 \\
(1.3)\end{array}$ & $\begin{array}{c}4.9 \\
(0.9)\end{array}$ & $\begin{array}{c}5.4 \\
(1.4)\end{array}$ & $\begin{array}{c}4.7 \\
(1.7)\end{array}$ & $\begin{array}{c}4.5 \\
(1.6)\end{array}$ & $\begin{array}{c}4.5 \\
(1.7)\end{array}$ & $\begin{array}{c}5.1 \\
(1.5)\end{array}$ \\
\hline $\begin{array}{l}\text { UE5. } \\
\text { Appeal. }\end{array}$ & $\begin{array}{c}4.2 \\
(1.3)\end{array}$ & $\begin{array}{c}5.3 \\
(1.1)\end{array}$ & $\begin{array}{c}4.1 \\
(1.5)\end{array}$ & $\begin{array}{l}5.5 \\
(1.0)\end{array}$ & $\begin{array}{c}5.1 \\
(1.1)\end{array}$ & $\begin{array}{c}5.7 \\
(0.8)\end{array}$ & $\begin{array}{c}3.8 \\
(1.5)\end{array}$ & $\begin{array}{c}5.2 \\
(1.3)\end{array}$ \\
\hline $\begin{array}{l}\text { UE6. } \\
\text { Recommend. }\end{array}$ & $\begin{array}{c}4.2 \\
(1.4)\end{array}$ & $\begin{array}{c}5.0 \\
(1.4)\end{array}$ & $\begin{array}{c}4.1 \\
(1.4)\end{array}$ & $\begin{array}{c}5.0 \\
(1.6)\end{array}$ & $\begin{array}{l}5.0 \\
(1.3)\end{array}$ & $\begin{array}{c}5.0 \\
(1.5)\end{array}$ & $\begin{array}{c}4.7 \\
(1.7)\end{array}$ & $\begin{array}{c}4.9 \\
(1.8)\end{array}$ \\
\hline $\begin{array}{l}\text { UE7. } \\
\text { Curious. }\end{array}$ & $\begin{array}{c}4.0 \\
(1.9)\end{array}$ & $\begin{array}{c}4.9 \\
(1.5)\end{array}$ & $\begin{array}{c}3.6 \\
(1.7)\end{array}$ & $\begin{array}{c}4.6 \\
(1.9)\end{array}$ & $\begin{array}{c}5.6 \\
(1.5)\end{array}$ & $\begin{array}{c}5.6 \\
(1.6)\end{array}$ & $\begin{array}{c}4.2 \\
(1.9)\end{array}$ & $\begin{array}{c}4.9 \\
(1.8)\end{array}$ \\
\hline $\begin{array}{l}\text { UE8. } \\
\text { Interested. }\end{array}$ & $\begin{array}{c}4.3 \\
(1.7)\end{array}$ & $\begin{array}{c}5.2 \\
(1.5)\end{array}$ & $\begin{array}{c}4.6 \\
(1.5)\end{array}$ & $\begin{array}{c}5.0 \\
(1.5)\end{array}$ & $\begin{array}{c}5.8 \\
(1.0)\end{array}$ & $\begin{array}{c}5.7 \\
(1.0)\end{array}$ & $\begin{array}{c}4.7 \\
(1.5)\end{array}$ & $\begin{array}{c}5.4 \\
(1.6)\end{array}$ \\
\hline $\begin{array}{l}\text { UE9. } \\
\text { Fun. }\end{array}$ & $\begin{array}{c}4.2 \\
(1.6)\end{array}$ & $\begin{array}{c}5.0 \\
(1.7)\end{array}$ & $\begin{array}{c}4.2 \\
(1.6)\end{array}$ & $\begin{array}{c}4.7 \\
(2.0)\end{array}$ & $\begin{array}{l}5.6 \\
(0.8)\end{array}$ & $\begin{array}{c}5.7 \\
(1.0)\end{array}$ & $\begin{array}{c}4.4 \\
(1.8)\end{array}$ & $\begin{array}{c}5.0 \\
(1.5)\end{array}$ \\
\hline $\begin{array}{l}\text { UE10. } \\
\text { Involved. }\end{array}$ & $\begin{array}{r}4.6 \\
(1.6) \\
\end{array}$ & $\begin{array}{c}5.3 \\
(0.8) \\
\end{array}$ & $\begin{array}{r}4.6 \\
(1.4) \\
\end{array}$ & $\begin{array}{r}5.7 \\
(1.0)\end{array}$ & $\begin{array}{r}5.2 \\
(1.2) \\
\end{array}$ & $\begin{array}{c}5.7 \\
(0.7) \\
\end{array}$ & $\begin{array}{r}4.3 \\
(1.8) \\
\end{array}$ & $\begin{array}{r}5.5 \\
(0.8) \\
\end{array}$ \\
\hline
\end{tabular}

Table 2. Mean (and s.d.) for the User Engagement Scale [1-7]. Rating pairs favorable to $V F$ are bold.

rapid pulse on page swap, but all noticing wheel effects. Weak noticeability is explained by rare use (recycle bin, page swap) or subtlety (launcher). Means for 67/77 contribution assessments of friction effects made by each participant reflected positive opinions $\left(\chi^{2}=40.7, \mathrm{p}<.0001\right)$. Negative assessments were most common in Game.

Comparison. Table 3 shows that all but one of the 20 direct comparisons favors variable friction: $\chi^{2}=14.4, \mathrm{p}<.001$. None of the participants expressed a strong preference for constant high friction in any of the questions.

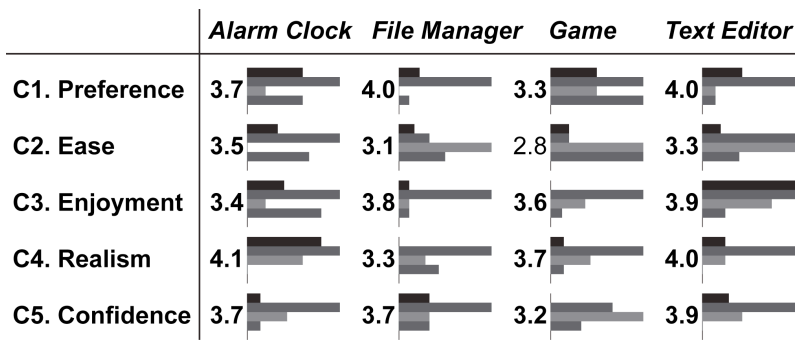

Table 3. Mean ratings [1-5] and distribution of answers (1 bottom - 5 top) for comparison questionnaire.

Final Questionnaire. Participant responses suggest that friction effects would not be turned off (mean 2.4, s.d. 1.0) and that they improved the experience (mean 4.1, s.d. 0.5 ).

\section{DISCUSSION AND NEXT STEPS}

We first investigated performance for variable friction effects and found measurable benefits without harm. S1 shows that variable friction (high on target, low elsewhere) significantly improves targeting performance. S2 verifies that variable friction is no worse than normal friction when distracters are present, crucial since pseudohaptic or haptic targets can decrease targeting performance in the presence of distracters $[6,14,20]$. S3 suggests that friction variations cause actual, not only perceived, velocity changes: unlike pseudohaptic and vibrotactile aids, friction slows the 
fingertip. Yet movement time does not rise, even with distracters, because approach stages are faster (S1-2).

We then focused on user experience, not performance [11] - engagement, enjoyment, directness, perceived utility and found through our S4 application samples that variable friction can enhance the emotional aspect of using a touch interface. This approach freed us to explore many design concepts and highlights what may ultimately be the most crucial factor in improving upon passive touch interfaces.

In the following we reflect upon generalizing these results to real-world use and understanding their value.

\section{Hardware Factors}

The LATPaD is currently a bulky prototype. However, the critical components for producing variable friction are the small, thin piezoelectric actuators visible in Figure 1a and there are no major barriers to miniaturizing the technology to the scale of current mobile devices. Rendering nonuniformities are also expected to be resolved in the near future, and were successfully avoided in our S4 designs.

\section{Performance in real-world target acquisition}

The LATPaD's variable friction effects are only felt when sliding against the display surface; S1-2 therefore used dragging tasks to maximally expose participants to the effects of interest. The lift-off selection used here is common on mobile devices, including the iPhone's text entry keypad. However, when approach occurs in the air, friction effects would only be felt during the final acquisition. This has been shown to reduce targeting performance with pen-based vibrotactile feedback [24]. We need to better understand how variable friction can benefit other selection modalities, but feel that first analyzing the most effective interaction for friction was appropriate.

\section{Variable Friction Versus Vibrotactile Feedback}

Vibrotactile (VT) feedback can provide selection performance improvements similar to variable friction, at least in single target conditions [24]. Variable friction and VT actuators, however, produce sensations that are very different. Friction tends to feel more natural and provides continuous feedback during sliding; vibrations are ideal for discrete clicks and textures, including tapping confirmation $[23,24]$. Variable friction can physically alter finger velocity, whereas VT can communicate a larger range of informative sensations [16], even without sliding. These two tactile modalities are complementary and in theory can be produced with the same actuators.

\section{Theoretical Design Space for Variable Friction}

The exemplar applications and widgets examined in S4 were developed through an iterative design process, and were successful in generating subjective responses. We took guidance both from the beginnings of our design space and from our intuition and iterations. We will refine this beginning with a taxonomy of variable friction sensations and their mapping to interactive widgets. This includes examining generalizations of our current set of widgets to other uses. For example, the Alarm Clock wheel was among the most popular, and we will examine how it can be deployed in support of related uses, such as scrolling. We will also investigate interaction techniques that minimize the use of low friction and hence actuator activation and power consumption, such as reduced friction on targets.

\section{Towards Variable Friction Design Heuristics}

In its infancy, the design of interactions with tactile feedback is prone to naive uses and excesses. We hope to launch a discussion of best practices for variable friction, extending a design space with heuristics such as these:

Sliding not tapping. To be effective, friction-augmented interfaces must work around the notion of sliding, with appropriate metaphors and visual representations. The Text Editor, for example, associated friction with word compression to give meaningful feedback during dragging.

Shaping friction to increase expressiveness. To compensate for the limited human sensitivity to friction variations and the current display range, expressiveness can be enhanced by varying friction 'attack', modulating it to create textures and patterns, and tying sensations to visual representations.

Stop only for a purpose. Some users felt that friction variations seemed occasionally to slow them down. Strong feedback should have an equally strong purpose.

Nice not strong. We tend to maximize tactile signal strength to ensure feedback is felt and performance improvements are measurable; but this can lead to unpleasant sensations.

\section{CONCLUSION}

Programmable friction displays vary the friction felt while sliding against a touch sensitive display. Through a series of studies and design explorations, we have demonstrated the strong potential of programmable friction interfaces. Most importantly, participants preferred our exemplar designs to traditional touch interactions and reported a variety of positive effects, including increased engagement, a sense of realism and reduced dependence on vision. In addition, our examination of programmable friction showed significant performance advantages for drag-based selections and no adverse effects when distracter targets are present.

This is the first analysis we are aware of for interaction with variable friction displays. These quantitative and qualitative results show exciting possibilities; the technology is on a development path 2-3 years from commercial realizability. There is great potential for more investigation: further performance analysis, design exploration and then deployment in mobile handhelds and laptop touchpads.

\section{REFERENCES}

1. Akamatsu, M. and MacKenzie, I.S. Movement characteristics using a mouse with tactile and force feedback. Int. J. of Human-Computer Studies 45, 4 (1996), 483-494. 
2. Balakrishnan, R. "Beating" Fitts' law: virtual enhancements for pointing facilitation. Int. J. of HumanComputer Studies 61, 6 (2004), 857-874.

3. Bau, O., Poupyrev, I., Israr, A., and Harrison, C. TeslaTouch: Electrovibration for Touch Surfaces. In Proc. UIST 2010, ACM (2010).

4. Benko, H., Wilson, A.D., and Balakrishnan, R. Sphere: Multi-Touch Interactions on a Spherical Display. In Proc. UIST 2008, ACM (2008), 77-86.

5. Buxton, W., Hill, R., and Rowley, P. Issues and techniques in touch-sensitive tablet input. In Proc. SIGGRAPH 1985, ACM (1985), 215-224.

6. Cockburn, A. and Brewster, S. Multimodal feedback for the acquisition of small targets. Ergonomics 48, 9 (2005), 1129-1150.

7. Dennerlein, J.T., Martin, D.B., and Hasser, C. Forcefeedback improves performance for steering and combined steering-targeting tasks. In Proc. CHI 2000, ACM (2000), 423-429.

8. DiFranco, D.E., Beauregard, G.L., and Srinivasan, M. The effect of auditory cues on the haptic perception of stiffness in virtual environments. In Proc. Haptics Symposium 1997, ASME (1997), 17-22.

9. Fitts, P.M. The information capacity of the human motor system in controlling the amplitude of movement. $J$. of Experimental Psychology 47, 6 (1954), 381-391.

10. Forlines, C. and Balakrishnan, R. Evaluating tactile feedback and direct vs. indirect stylus input in pointing and crossing selection tasks. In Proc. CHI 2008, ACM (2008), 1563-1572.

11. Greenberg, S. and Buxton, B. Usability evaluation considered harmful (some of the time). In Proc. CHI 2008, ACM (2008), 111-120.

12. Hancock, M., Carpendale, S., and Cockburn, A. Shallow-depth $3 \mathrm{~d}$ interaction: design and evaluation of one-, two- and three-touch techniques. In Proc. CHI 2007, ACM (2007), 1147-1156.

13. Hoggan, E., Kaaresoja, T., Laitinen, P., and Brewster, S. Crossmodal congruence: the look, feel and sound of touchscreen widgets. In Proc. IMCI 2008, ACM (2008), 157-164.

14. Keyson, D.V. Dynamic cursor gain and tactual feedback in the capture of cursor movements. Ergonomics 40, 12 (1997), 1287-1298.

15.Li, K.A., Baudisch, P., Griswold, W.G., and Hollan, J.D. Tapping and rubbing: exploring new dimensions of tactile feedback with voice coil motors. In Proc. UIST 2008, ACM (2008), 181-190.

16. MacLean, K.E. Foundations of Transparency in Tactile Information Design. IEEE Transactions on Haptics 1, 2 (2008), 84-95.
17. Marchuk, N.D., Colgate, J.E., and Peshkin, M.A. Friction measurements on a Large Area TPaD. In Proc. Haptics Symposium 2010, IEEE (2010), 317-320.

18. Moscovich, T. Contact area interaction with sliding widgets. In Proc. UIST 2009, ACM (2009), 13-22.

19. Norman, D. Emotional Design: Why We Love (or Hate) Everyday Things. Basic Books, NY, NY, USA, 2004.

20. Oakley, I., Brewster, S., and Gray, P. Solving multitarget haptic problems in menu interaction. In Proc. CHI 2001, ACM (2001), 357-358.

21. O'Brien, H.L. and Toms, E.G. The development and evaluation of a survey to measure user engagement. Journal of the American Society for Information Science and Technology 61, 1 (2009), 50-69.

22. Potter, R.L., Weldon, L.J., and Shneiderman, B. Improving the accuracy of touch screens: an experimental evaluation of three strategies. In Proc. CHI 1988, ACM (1988), 27-32.

23. Poupyrev, I. and Maruyama, S. Tactile interfaces for small touch screens. In Proc. UIST 2003, ACM (2003), 217-220.

24. Poupyrev, I., Okabe, M., and Maruyama, S. Haptic feedback for pen computing: directions and strategies. In Proc. CHI 2004, ACM (2004), 1309-1312.

25. Ramos, G. and Balakrishnan, R. Fluid interaction techniques for the control and annotation of digital video. In Proc. UIST 2003, ACM (2003), 105-114.

26. Rekimoto, J. Organic interaction technologies. Communications of the ACM 51, 6 (2008), 38-44.

27. Roudaut, A., Huot, S., and Lecolinet, E. TapTap and MagStick: improving one-handed target acquisition on small touch-screens. In Proc. AVI 2008, ACM (2008), 146-153.

28. Schiphorst, T., Motamedi, N., and Jaffe, N. Applying an Aesthetic Framework of Touch for Table-Top Interactions. In Proc. TABLETOP 2007, IEEE (2007), 71-74.

29. Shneiderman, B. Direct Manipulation: A Step Beyond Programming Languages. Computer 16, 8 (1983), 5769.

30. Smyth, T.N. and Kirkpatrick, A.E. A new approach to haptic augmentation of the GUI. In Proc. ICMI 2006, ACM (2006), 372-379.

31. Swindells, C., MacLean, K.E., Booth, K.S., and Meitner, M.J. Exploring affective design for physical controls. In Proc. CHI 2007, ACM (2007), 933-942.

32. Winfield, L., Glassmire, J., Colgate, J.E., and Peshkin, M. T-PaD: Tactile Pattern Display through Variable Friction Reduction. In Proc. World Haptics 2007, IEEE (2007), 421-426. 